\title{
ON EXTREME RUINOUS BEHAVIOUR OF LÉVY INSURANCE RISK PROCESSES
}

\author{
C. KLÜPPELBERG, ${ }^{*}$ Munich University of Technology \\ A. E. KYPRIANOU, ${ }^{* *}$ Heriot-Watt University
}

\begin{abstract}
In this short note we show how new fluctuation identities and their associated asymptotics, given in Vigon (2002), Klüppelberg et al. (2004) and Doney and Kyprianou (2006), provide the basis for establishing, in an elementary way, asymptotic overshoot and undershoot distribitions for a general class of Lévy insurance risk processes. The results bring the earlier conclusions of Asmussen and Klüppelberg (1996) for the CramérLundberg process into greater generality.
\end{abstract}

Keywords: Lévy process; insurance risk process; ruin; extreme value theory

2000 Mathematics Subject Classification: Primary 60K05; 60K15; 91B30

Secondary 60G70; 60J55

\section{Lévy processes and the structure of insurance claims}

Recall that the Cramér-Lundberg model corresponds to a Lévy process

$$
X^{\mathrm{CL}}=\left\{X_{t}^{\mathrm{CL}}: t \geq 0\right\}
$$

with characteristic exponent given by

$$
\Psi^{\mathrm{CL}}(\theta)=-\log \int_{\mathbb{R}} \mathrm{e}^{\mathrm{i} \theta x} \mathrm{P}\left(X_{1}^{\mathrm{CL}} \in \mathrm{d} x\right)=-\mathrm{i} c \theta+\lambda \int_{(0, \infty)}\left(1-\mathrm{e}^{\mathrm{i} \theta x}\right) F(\mathrm{~d} x),
$$

for $\theta \in \mathbb{R}$ such that $\lim _{t \uparrow \infty} X_{t}^{\mathrm{CL}}=\infty$. In other words, $X^{\mathrm{CL}}$ is a compound Poisson process with arrival rate $\lambda>0$ and negative jumps, corresponding to claims, having common distribution function $F$ with finite mean $\mu$, as well as a drift $c>0$, corresponding to a steady income due to premiums, which necessarily satisfies $c-\lambda \mu>0$. Suppose instead that we work with a general spectrally negative Lévy process, that is, a Lévy process

$$
X^{\mathrm{SN}}=\left\{X_{t}^{\mathrm{SN}}: t \geq 0\right\}
$$

that drifts to infinity with Lévy measure $\Pi$ satisfying $\Pi(0, \infty)=0$. Such processes have been considered recently by Huzak et al. (2004a), (2004b) and Klüppelberg et al. (2004) in the context of insurance risk models. In this case, the Lévy-Itô decomposition offers an interpretation for

Received 7 September 2005; revision received 15 December 2005.

* Postal address: Center for Mathematical Sciences, Munich University of Technology, Boltzmannstrasse 3, D-85747 Garching, Germany. Email address: cklu@ma.tum.de

** Postal address: School of Mathematical and Computer Sciences, Heriot-Watt University, Edinburgh EH14 4AS, UK. Email address: kyprianou@ma.hw.ac.uk 
large-scale insurance companies as follows. The characteristic exponent may be written in the form

$$
\begin{aligned}
\Psi^{\mathrm{SN}}(\theta)= & -\log \int_{\mathbb{R}} \mathrm{e}^{\mathrm{i} \theta x} \mathrm{P}\left(X_{1}^{\mathrm{SN}} \in \mathrm{d} x\right) \\
= & \left\{\frac{1}{2} \sigma^{2} \theta^{2}\right\}+\left\{-\mathrm{i} \theta c+\int_{(-\infty,-1)}\left(1-\mathrm{e}^{\mathrm{i} \theta x}\right) \Pi(\mathrm{d} x)\right\} \\
& +\left\{\int_{(-1,0)}\left(1-\mathrm{e}^{\mathrm{i} \theta x}+\mathrm{i} \theta x\right) \Pi(\mathrm{d} x)\right\},
\end{aligned}
$$

for $\theta \in \mathbb{R}$; necessarily $\sigma^{2} \geq 0$ and the Lévy measure, $\Pi$, satisfies $\int_{(-\infty, 0)}\left(1 \wedge x^{2}\right) \Pi(\mathrm{d} x)<\infty$. The requirement that $X$ drifts to infinity implies that $c-\int_{(-\infty,-1)}|x| \Pi(\mathrm{d} x)>0$. Note that when $\Pi(-\infty, 0)=\infty$ the process $X^{\mathrm{SN}}$ enjoys a countably infinite number of jumps over each finite time horizon. We may understand the third bracket in (1) as a Lévy process representing a countably infinite number of arbitrarily small claims compensated by a deterministic positive drift (which may be infinite in the case that $\int_{(-1,0)}|x| \Pi(\mathrm{d} x)=\infty$ ) corresponding to the accumulation of premiums over an infinite number of contracts. Roughly speaking, the way in which claims occur is such that, in any arbitrarily small period of time $\mathrm{d} t$, a claim of size $|x|$ is made independently with probability $\Pi(\mathrm{d} x) \mathrm{d} t+o(\mathrm{~d} t)$. The insurance company thus counterbalances such claims by ensuring that it collects premiums in such a way that, in any $\mathrm{d} t$, $|x| \Pi(\mathrm{d} x) \mathrm{d} t$ of its income is devoted to the compensation of claims of size $|x|$. We may understand the second bracket in (1) as coming from large claims which occur occasionally and are compensated against by a steady income at rate $c>0$ as in the Cramér-Lundberg model. Here 'large' is taken to mean claims of size one or more. Finally, we may see the first bracket in (1) as a stochastic pertubation of the system of claims and premium income.

Since the first and third brackets in (1) correspond to martingales, the company may guarantee that its revenues drift to infinity over an infinite time horizon by assuming that the latter behaviour applies to the compensated process of large claims corresponding to the second bracket in (1).

\section{Extreme ruinous behaviour}

In this short note our objective is to show that, thanks to the recent results of Vigon (2002), Klüppelberg et al. (2004), and Doney and Kyprianou (2006), conclusions to be found in Asmussen and Klüppelberg (1996) concerning the extreme ruinous behaviour under assumptions of subexponentiality in the jump distribution of the classic Cramér-Lundberg model can be extended effortlessly to the case of a general spectrally negative process. In the usual way we turn the problem around and consider the first passage of the dual process above a fixed barrier. In that case we deal with the process $X=\left\{X_{t}: t \geq 0\right\}$ such that, under $\mathrm{P}, X$ has the same law as $-X^{\mathrm{SN}}$. Note in particular that now $\lim _{t \uparrow \infty} X_{t}=-\infty$ and, hence, from the discussion in Section 1 we necessarily have $-\infty<\mathrm{E}\left(X_{1}\right)<0$. We shall denote the Lévy measure of $X$ by $\Pi_{X}$

Recall that a distribution function $F$ supported on $[0, \infty)$ is subexponential if $\bar{F}(x):=$ $1-F(x)>0$ for each $x \geq 0$ and, furthermore, the tail of the two-fold convolution satisfies

$$
\lim _{x \uparrow \infty} \frac{\overline{F^{* 2}}(x)}{\bar{F}(x)}=2 .
$$

The definition of subexponentiality can be extended to any measure with positive support which is finite on $\left(x_{0}, \infty\right)$ for some $x_{0} \geq 0$ (for example a Lévy measure) by normalizing it to 
a probability measure. There is a very broad literature concerning subexponential distributions and their applications. We refer to Embrechts et al. (1997, Chapter 1 and Appendix A3) or Goldie and Klüppelberg (1998) for overviews.

A classic result due to Embrechts et al. (1979) shows that if $F$ is an infinitely divisible distribution on $[0, \infty)$, then $F$ is subexponential if and only if $\Pi_{F}$ is subexponential, where $\Pi_{F}$ is the Lévy measure appearing in the Lévy-Khinchin decomposition associated with $F$. Furthermore, in that case, $\bar{\Pi}_{F}(x) \sim \bar{F}(x)$ as $x \uparrow \infty$.

In our two main results below the conditions on $X$ shall be sufficient to deduce that the Lévy measure of the ascending ladder process, $\Pi_{H}$, belongs to the subexponential class. In that case, Lemma 3.5 of Klüppelberg et al. (2004) shows that the probability of ruin has the asymptotic behaviour

$$
\lim _{x \uparrow \infty} \frac{\mathrm{P}\left(\sigma_{x}^{+}<\infty\right)}{\bar{\Pi}_{H}(x)}=\frac{1}{\left|\mathrm{E}\left(X_{1}\right)\right|},
$$

where $\sigma_{x}^{+}=\inf \left\{t>0: X_{t}>x\right\}$ and $\bar{\Pi}_{H}(x)=\Pi_{H}(x, \infty)$.

\subsection{Regularly varying tails}

Let $\mathcal{R}^{(\alpha)}$ be the class of functions which are regularly varying with index $\alpha$. That is, we say that $f \in \mathcal{R}^{(\alpha)}$ if $f$ is a measurable and positive function on $(0, \infty)$ and, for all $\lambda>0$,

$$
\lim _{x \uparrow \infty} \frac{f(x \lambda)}{f(x)}=\lambda^{\alpha}
$$

(cf. Embrechts et al. (1997, Appendix A3)).

Theorem 1. Suppose that $X$ is any spectrally positive Lévy process which drifts to $-\infty$, and suppose that $\bar{\Pi}_{X}(\cdot) \in \mathcal{R}^{(-\alpha-1)}$ for some $\alpha \in(0, \infty)$. Then the following asymptotic bivariate law holds as $x \rightarrow \infty$ :

$$
\left(\frac{-X_{\sigma_{x}^{+}-}}{a(x)}, \frac{X_{\sigma_{x}^{+}}-x}{a(x)}\right) \rightarrow\left(V_{\alpha}, T_{\alpha}\right)
$$

in $\mathrm{P}\left(\cdot \mid \sigma_{x}^{+}<\infty\right)$-distribution in $\mathbb{R} \times \mathbb{R}_{+}$, where $a(x)=x / \alpha$ and the pair $\left(V_{\alpha}, T_{\alpha}\right)$ are dependent Pareto random variables satisfying

$$
\mathrm{P}\left(V_{\alpha}>x, T_{\alpha}>y\right)=\left(1+\frac{x+y}{\alpha}\right)^{-\alpha}, \quad x, y>0 .
$$

Proof. Let $U(\mathrm{~d} x)$ be the potential measure of the ascending ladder height process $\left\{H_{t}: t \geq 0\right\}$, where $H_{t}=X_{L_{t}^{-1}}$ for $t<L_{\infty}$ and $\left\{L_{t}: t \geq 0\right\}$ is the Markov local time spent at zero of the process $\left\{\sup _{s \leq t} X_{s}-X_{t}: t \geq 0\right\}$. Hence, $U(\mathrm{~d} x)=\mathrm{E}\left(\int_{0}^{\infty} \mathbf{1}_{\left\{H_{t} \in \mathrm{d} x\right\}} \mathrm{d} t\right)$, where $\mathbf{1}_{\{.\}}$is the indicator function. We write $U(x)=U([0, x])$. Then it is also known that $U(x)$ is the continuous function identifiable by its Laplace transform $\int_{0}^{\infty} \mathrm{e}^{-\beta x} U(x) \mathrm{d} x=1 / \psi(\beta)$ for $\beta>0$, where $\psi(\beta)=\log \mathrm{E}\left(\mathrm{e}^{-\beta X_{1}}\right)$. It is also known (cf. Klüppelberg et al. (2004, Remark 4.3)) that $U(\infty)=1 /\left|\mathrm{E}\left(X_{1}\right)\right|$.

As a special case of the quintuple law given in Theorem 3 of Doney and Kyprianou (2006), we may now write, for $u^{*}, v^{*}>0$,

$$
\mathrm{P}\left(X_{\sigma_{x}^{+}}-x>u^{*}, x-X_{\sigma_{x}^{+}-}>v^{*}\right)=\int_{0}^{x} U(x-\mathrm{d} y) \int_{\left[v^{*} \vee y, \infty\right)} \mathrm{d} z \int_{\left[u^{*}, \infty\right)} \Pi_{X}(\mathrm{~d} u+z) .
$$


Consequently, setting $v^{*}=x+a(x) v$ and $u^{*}=a(x) u$ and taking into account the relation between $\Pi_{H}$ and $\Pi_{X}$ established by Vigon (2002), namely

$$
\bar{\Pi}_{H}(u)=\int_{u}^{\infty} \bar{\Pi}_{X}(z) \mathrm{d} z, \quad u>0,
$$

we have

$$
\mathrm{P}\left(\frac{X_{\sigma_{x}^{+}}-x}{a(x)}>u, \frac{-X_{\sigma_{x}^{+}-}}{a(x)}>v\right)=U(x) \bar{\Pi}_{H}(x+a(x)(v+u)) .
$$

The assumption that $\bar{\Pi}_{X}(\cdot) \in \mathcal{R}^{(-1-\alpha)}$ and the relation (4) imply (by Karamata's theorem; see Embrechts et al. (1997, Section A3)) that the integrated tail $\bar{\Pi}_{H}(x)$ is in $\mathcal{R}^{(-\alpha)}$. In particular, this means that $\Pi_{H}$ belongs to the subexponential class. We now have

$$
\begin{aligned}
\lim _{x \uparrow \infty} \mathrm{P} & \left(\frac{X_{\sigma_{x}^{+}}-x}{a(x)}>u, \frac{-X_{\sigma_{x}^{+}-}}{a(x)}>v \mid \sigma_{x}^{+}<\infty\right) \\
\quad= & \lim _{x \uparrow \infty}\left|\mathrm{E}\left(X_{1}\right)\right| U(x) \frac{\bar{\Pi}_{H}(x+a(x)(v+u))}{\bar{\Pi}_{H}(x)} \\
\quad= & \left(1+\frac{v+u}{\alpha}\right)^{-\alpha},
\end{aligned}
$$

where we have appealed to (2) and regular variation in the first equality and second equality, respectively. This is consistent with the statement of the theorem.

\subsection{Maximum domain of attraction of the Gumbel distribution}

Recall that a distribution function $F$ is in the maximal domain of attraction of the Gumbel distribution if and only if there exists a positive differentiable function $a(\cdot)$ satisfying $a^{\prime}(x) \rightarrow 0$ such that

$$
\lim _{x \uparrow \infty} \frac{\bar{F}(x+a(x) u)}{\bar{F}(x)}=\mathrm{e}^{-u},
$$

for all $u>0$ (see, e.g. Embrechts et al. (1997, Theorems 3.3.26 and 3.3.27)). A possible choice of $a$ is

$$
a(x)=\int_{x}^{\infty} \frac{\bar{F}(z)}{\bar{F}(x)} \mathrm{d} z
$$

where $a$ is unique only up to asymptotic equivalence. Recall that for $F$ infinitely divisible with Lévy measure $\Pi_{F}$ we know that $\bar{F}(x) \sim \bar{\Pi}_{F}(x)$ as $x \uparrow \infty$. As any maximum domain of attraction is closed with respect to tail equivalence we may also say that $\Pi_{F}$ is in the maximal domain of attraction of a Gumbel distribution.

Theorem 2. Suppose that $X$ is any spectrally positive Lévy process which drifts to $-\infty$, and suppose that $\bar{\Pi}_{X}$ is in the maximum domain of attraction of the Gumbel distribution. Then the asymptotic bivariate law (3) holds as $x \rightarrow \infty$, where $a(x) \sim \int_{x}^{\infty} \bar{\Pi}_{X}(z) \mathrm{d} z / \bar{\Pi}_{X}(x)$ as $x \rightarrow \infty$ and the pair $\left(V_{\alpha}, T_{\alpha}\right)$ are dependent exponential random variables satisfying

$$
\mathrm{P}\left(V_{\alpha}>x, T_{\alpha}>y\right)=\mathrm{e}^{-(x+y)}, \quad x, y>0 .
$$

Proof. Following the previous proof, we pick up at (5). The assumption on $\bar{\Pi}_{X}$ implies that $a^{\prime}(x) \rightarrow 0$ as $x \rightarrow \infty$ and

$$
\frac{\bar{\Pi}_{X}(x+a(x)(v+u))}{\bar{\Pi}_{X}(x)} \rightarrow \mathrm{e}^{-(v+u)}, \quad x \rightarrow \infty .
$$


The proof is complete once it is shown that a similar relation holds for $\bar{\Pi}_{H}(x)=\int_{x}^{\infty} \bar{\Pi}_{X}(y) \mathrm{d} y$. To show that this is indeed the case, we use l'Hôpital's rule:

$$
\lim _{x \rightarrow \infty} \frac{\bar{\Pi}_{H}(x+a(x)(v+u))}{\bar{\Pi}_{H}(x)}=\lim _{x \rightarrow \infty} \frac{\bar{\Pi}_{X}(x+a(x)(v+u))\left(1+a^{\prime}(x)\right)}{\bar{\Pi}_{X}(x)}=\mathrm{e}^{-(v+u)}
$$

as $a^{\prime}(x) \rightarrow 0$.

\section{Concluding remark}

Note that further results follow from the limiting bivariate law in Asmussen and Klüppelberg (1996) (for example their Corollary 1.5), which, given the conclusions of Theorems 1 and 2, have direct analogues within the current context, with identical proofs.

\section{References}

Asmussen, S. ANd KlüPPelberg, C. (1996). Large deviations results for subexponential tails, with applications to insurance risk. Stoch. Process. Appl. 64, 103-125.

Doney, R. A. and Kyprianou, A. E. (2006). Overshoots and undershoots of Lévy processes. Ann. Appl. Prob. 16, 91-106.

Embrechts, P., Goldie, C. M. and VeraverbeKe, N. (1979). Subexponentiality and infinite divisibility. Z. Wahrscheinlichkeitsth. 49, 335-347.

Embrechts, P., Klüppelberg, C. ANd Mikosch, T. (1997). Modelling Extremal Events for Insurance and Finance. Springer, Berlin.

Goldie, C. M. And KlüPpelberg, C. (1998). Subexponential distributions. In A Practical Guide to Heavy Tails, eds R. Adler, R. Feldman and M. S. Taqqu, Birkhäuser, Boston, MA, pp. 435-459.

Huzak, M., Perman, M., ŠIKIĆ, H. AND Vondraček, Z. (2004a). Ruin probabilities and decompositions for general perturbed risk processes. Ann. Appl. Prob. 14, 1378-1397.

HuZak, M., Perman, M., ŠIKIĆ, H. ANd VondraČEK, Z. (2004b). Ruin probabilities for competing claim processes. J. Appl. Prob. 41, 679-690.

Klüppelberg, C., Kyprianou, A. E. And Maller, R. (2004). Ruin probabilities and overshoots for general Lévy insurance risk processes. Ann. Appl. Prob. 14, 1766-1801.

Vigon, V. (2002). Votre Lévy rampe-t-il? J. London Math. Soc. 65, 243-256. 